\title{
Relationships of Sleep Duration, Midpoint, and Variability with Physical Activity in the HCHS/SOL Sueño Ancillary Study
}

\author{
Kimberly L. Savin (ID), Sanjay R. Patel ${ }^{b}$, Taylor L. Clarka, Julia I. Bravina , Scott C. Roesch', \\ Daniela Sotres-Alvarez ${ }^{\mathrm{d}}$, Yasmin Mossavar-Rahmani ${ }^{\mathrm{e}}$, Kelly R. Evenson ${ }^{\mathrm{f}}$, Martha Daviglus ${ }^{\mathrm{g}}$, \\ Alberto R. Ramos ${ }^{\text {, }}$ Phyllis C. Zee', Marc D. Gellman', and Linda C. Galloc \\ aSan Diego Joint Doctoral Program in Clinical Psychology, San Diego State University/University of California, San \\ Diego, California; bepartment of Medicine, University of Pittsburgh School of Medicine, Pittsburgh, Pennsylvania; \\ 'Department of Psychology, San Diego State University, San Diego, California; ${ }^{d}$ Department of Biostatistics, Gillings \\ School of Global Public Health, University of North Carolina at Chapel Hill, Chapel Hill, North Carolina; 'Department of \\ Epidemiology \& Population Health, Albert Einstein College of Medicine, Bronx, New York; 'Department of \\ Epidemiology, Gillings School of Global Public Health, University of North Carolina at Chapel Hill, Chapel Hill, North \\ Carolina; ${ }^{9}$ Department of Preventive Medicine, University of Illinois at Chicago, Chicago, Illinois; ${ }^{\text {hDepartment of }}$ \\ Neurology, University of Miami, Miami, Florida; 'Department of Neurology, Northwestern University Feinberg School of \\ Medicine, Chicago, Illinois; iDepartment of Psychology, University of Miami, Miami, Florida
}

\begin{abstract}
Objective/Background: Short and long sleep duration, later sleep midpoint, and greater intra-individual sleep variability are associated with lower physical activity, but previous research lacks objective and concurrent assessment of sleep and physical activity. This cross-sectional study examined whether sleep duration, midpoint, and variability in duration and midpoint were related to wrist actigraphy-measured physical activity.

Participants: Participants were 2156 Hispanics/Latinos in the Hispanic Community Health Study/Study of Latinos (HCHS/SOL) Sueño Ancillary Study.

Methods: Participants wore Actiwatch devices to measure sleep and physical activity via the wrist for $\geq 5$ days. Physical activity was defined as minutes/day in the upper quartile of the sampling distribution's non-sleep activity, capturing light to vigorous physical activity.

Results: An inverse linear relationship between sleep duration and physical activity was found such that each additional sleep hour related to 29 fewer minutes of physical activity $(B=-28.7, \mathrm{SE}=3.8), p<.01)$. Variability in sleep midpoint was also associated with physical activity; with each 1-hr increase in variability there were 24 more minutes of physical activity $(B=24.2, S E=5.6$, $p<.01)$. In contrast, sleep midpoint and variability in duration were not associated with physical activity. Sensitivity analyses identified an association of short sleep duration and greater variability in sleep duration with greater accelerometry-derived moderate-to-vigorous physical activity measured at the $\mathrm{HCHS} / \mathrm{SOL}$ baseline ( $\mathrm{M}=2.1$ years before the sleep assessment).

Conclusions: Findings help clarify inconsistent prior research associating short sleep duration and sleep variability with greater health risks but also contribute novel information with simultaneous objective assessments.
\end{abstract}

\section{Introduction}

Sleep duration and timing influence risk of obesity, diabetes, and other cardiometabolic disorders in prospective studies (Ogilvie \& Patel, 2017; Shan et al., 2015). While some research suggests physiological factors (i.e., the circadian rhythm's role in regulation of appetite and stress hormones and 
inflammatory proteins) may underlie these relationships (Knutson et al., 2007; Wright et al., 2015), time spent engaging in healthy behaviors, such as physical activity may also be an important mechanism in the pathway from sleep to health.

Most of the research surrounding sleep and health behaviors has focused on sleep duration. A curvilinear relationship between sleep duration and objectively measured physical activity has been observed in the National Health and Nutrition Survey (NHANES), such that both short and long sleep duration were linked with less physical activity; however, sleep duration was measured by self-report with its inherent limitations (McClain et al., 2014). In another study examining the associations of self-reported sleep duration and physical activity, obtaining $>6-7 \mathrm{hr}$ of sleep was associated with greater odds of meeting physical activity guidelines compared to $\leq 6 \mathrm{hr}$, but associations between sleep and physical activity were not significant when comparing groups with greater levels of sleep duration to those who obtained $\leq 6 \mathrm{hr}$ (Towne et al., 2017). Longer self-reported sleep duration has been associated with less self-reported (Bellavia et al., 2014) and objectively measured physical activity (Gabriel et al., 2017). Specifically, longer self-reported sleep ( $>9 \mathrm{hr}$ ) was associated with lower daily total activity and a reduced likelihood of achieving $\geq 20 \mathrm{~min}$ of moderate-to-vigorous physical activity (MVPA; Gabriel et al., 2017). Sleep timing and the midpoint of sleep also have been associated with physical activity in several studies. Later sleep timing has been linked with less physical activity (Rutters et al., 2014), but sleep was measured by self-report. In a small study that examined sleep and physical activity using wrist actigraphy, later sleep midpoint was associated with less light intensity physical activity and MVPA (Shechter \& St-Onge, 2014). Few studies have examined objective measures of sleep timing in relation to physical activity in large adult samples. Given the unclear pattern of findings in studies relying mainly on self-report approaches and small sample sizes, further investigation using objective sleep and activity measures and larger samples is needed to better understand these associations.

Sleep variability, operationalized as the intra-individual variability in sleep characteristics (e.g., duration, midpoint) across environmental contexts (e.g., from a work night to a free night), may also have health implications. Variability partially reflects social "jetlag," a phenomenon occurring when individuals go to sleep and wake up later on free nights than they do on work or school nights (Wittman et al., 2006), or due to differing work schedules (e.g., shift work; Reid et al., 2018). The shift to accommodate social and occupational activities leads to disruptions in circadian rhythms, which have negative implications for cardiometabolic health indicators such as impaired glucose tolerance and insulin resistance in experimental studies (Morris et al., 2015; Wright et al., 2015). Both greater variability in self-reported bed times and wake times (Duncan et al., 2016) and greater differences in self-reported sleep midpoint between work days and free days (Rutters et al., 2014) have been associated with less physical activity. Further research using objective measurement of sleep and physical activity is needed to better understand these associations.

Limited research has examined relationships between sleep duration, variability, and physical activity, particularly in Hispanics/Latinos, who are at increased risk for low levels of physical activity (Arredondo et al., 2016) and poorer sleep health (Dudley et al., 2017). Hispanics/Latinos face unique stressors (i.e., ethnic discrimination, acculturation stress) that have been shown to influence sleep (Alcántara et al., 2017). A previous study in this cohort found that self-reported short sleep duration ( $<7 \mathrm{hr}$ ) was associated with $26 \mathrm{~min}$ /day more of work-related (e.g., paid work, household chores) physical activity (Tom et al., 2019). Similarly, another study in Hispanics/Latinos showed that the nature of the association differed by physical activity type, with caregiving activities relating to a greater likelihood of short sleep, and light intensity physical activity to a lower likelihood of long sleep (Hawkins et al., 2018).

The current study addresses gaps in the literature by examining cross-sectional relationships of objectively and concurrently measured sleep duration and physical activity among Hispanic/Latino adults 18-64 years of age. The current study also examined the additional associations of variability in nightly sleep duration and sleep midpoint, over and above mean sleep duration and midpoint, with physical activity. We hypothesized that shorter and longer sleep duration (compared to intermediate 
duration), later sleep midpoint, and greater variability in both sleep duration and midpoint would be associated with lower levels of physical activity.

\section{Methods}

\section{Study design}

The Hispanic Community Health Study/Study of Latinos (HCHS/SOL; 2008-2011) is a populationbased cohort of 16,415 self-identified Hispanics/Latino adults from four urban US communities (Bronx, NY; Chicago, IL; Miami, FL; and San Diego, CA). Participants were enrolled using a stratified 2-stage area probability sampling design (LaVange et al., 2010; Sorlie et al., 2010). This design allowed for a broadly diverse sample, socioeconomic status, and age distribution. Sampling weights were adopted to account for probability of selection and nonresponse to ensure that the estimates from the study would be representative of the target populations (LaVange et al., 2010). The Sueño ancillary study enrolled a subset of 2,252 participants from HCHS/SOL (2010-2013) who were within 30 months of their baseline visit, between 18 and 64 years of age, and free of narcolepsy, severe obstructive sleep apnea, and use of nocturnal positive airway pressure therapy (Patel et al., 2015). All participants gave written consent and the protocol was approved by the institutional review boards for all participating institutions. Of those enrolled, 2,156 participants (96\%) had at least 5 valid days of actigraphy data (considered invalid if $>4 \mathrm{hr}$ of missing data or $>2$ minutes of missing data during sleep in main rest interval; Patel et al., 2015) and were included in the current analytic sample.

\section{Study procedure}

The current study used cross-sectional objective sleep and physical activity from the Sueño assessment, and objective physical activity data and sociodemographic information from the HCHS/SOL baseline visit, as described next.

\section{Primary variable measures}

\section{Sleep}

In the Sueño assessment, each participant received an Actiwatch Spectrum (Philips Respironics, Murrysville, PA) to be worn on the non-dominant wrist for $\geq 5$ days and nights, and also completed a brief daily sleep diary. Sleep data were processed using a standardized reproducible scoring algorithm, integrating both diary and raw sensor data (Patel et al., 2015). Sleep duration in hours was the total time scored in 30 second epochs as sleep between sleep onset and offset during the main sleep period. In categorical analysis of sleep duration, short, intermediate, and long sleep were defined as $<6 \mathrm{hr}, 6-8 \mathrm{hr}$, and $>8 \mathrm{hr}$, respectively (Mossavar-Rahmani et al., 2017). Sleep midpoint represents the clock time midway between sleep onset and offset. The current study defined sleep midpoint continuously, as the number of minutes after 4:00 AM (approximate median midpoint of the sample). Within-person sleep variability was the standard deviation (SD) of a participant's nightly sleep duration and sleep midpoint.

\section{Physical activity}

Physical activity data were also obtained from the Actiwatch. Data collected by the Actiwatch during active (i.e., non-sleep) epochs of 30-s duration were categorized into physical activity according to their number of accelerations (in activity counts) per minute to mirror more standardized physical activity measures/recommendations (e.g., 2018 Physical Activity Guidelines Advisory Committee, 2018). There is little research establishing and validating cutpoints to determine physical activity from the wrist-worn device used in the current study. We used a cutoff value at the upper quartile (Murillo et al., 2017; defined as $\geq 245$ counts/30-s epoch, equivalent to 490 counts/min) of the sampling 
distribution, which captured light to vigorous physical activity. A sensitivity analysis was conducted examining the average activity counts/minute during all active (non-rest) intervals.

Additional sensitivity analyses were conducted using physical activity information from the hip-worn accelerometer data collected at the HCHS/SOL baseline visit (Actical version B-1 model 198-0200-03; Respironics Co. Inc., Bend, OR). Participants were instructed to wear the device for 1 week during waking hours. This device captured physical activity counts in 1-min epochs and has well-established cutoff values (Colley \& Tremblay, 2011) which have previously been used in this cohort (Evenson et al., 2015). Epochs with activity count $\geq 1535$ counts/min were considered MVPA (Colley \& Tremblay, 2011). Because the baseline assessment data were collected an average of 2.1 years ( $\mathrm{SD}=0.4$ years) before the Sueño assessment, and the aim of this study was to examine contemporaneous associations of sleep and physical activity, these baseline data were only used in sensitivity analyses.

\section{Covariate measures}

\section{Sociodemographic characteristics}

Sociodemographic information, including age, sex, field center, household income, and employment status, was obtained via interview administered self-report at the Sueño assessment, where available, or the HCHS/SOL baseline visit.

\section{Depression}

Depression symptoms were measured at the Sueño assessment with the Center for Epidemiologic Studies Depression Scale 10 (CES-D-10; Andresen et al., 1994). This 10-item screening tool measured self-reported depression symptoms in the past week, and has been tested and established as reliable and valid in the HCHS/SOL cohort (González et al., 2017).

\section{Comorbid health conditions}

Potentially confounding physically limiting conditions were assessed using a summative comorbidity index [range from 0 to 4] in which one point was scored for having diabetes, cardiovascular disease, obstructive lung disease, and cancer history, as self-reported or measured at baseline (Vásquez et al., 2016).

\section{Statistical analysis}

Analyses were performed using IBM SPSS Statistics Version 24 (IBM, Inc., Armonk, NY) and Mplus Version 7.31 (Muthén \& Muthén, 2006) and accounted for HCHS/SOL complex survey design and Sueño sampling weights to account for non-response to the ancillary study. In primary analyses, we fit a multivariable linear regression model with continuous sleep duration as an explanatory variable and physical activity, indicated as the upper quartile of Actiwatch epochs, as the response variable (model 1). Next, variability in sleep duration was added as an explanatory variable to the model (model 2). A similar model with categorical sleep duration variables was fit to test for potential curvilinear relationships (model 3). Model 4 examined sleep midpoint as the explanatory variable and variability of sleep midpoint was added as an explanatory variable in model 5. All analyses controlled for the complex survey design and sampling weights, sociodemographic (i.e., age, sex, field center, income, and employment status), and health characteristics (i.e., depression and comorbidities, as described earlier). Further, the two sleep variability models included continuous sleep duration or sleep midpoint as covariates to determine the influence of sleep variability over and above average duration or midpoint.

Several sensitivity analyses were conducted. First, we repeated analyses examining the average activity counts/minute during all active (non-rest) intervals as an alternative outcome for the Actiwatch. We found that the correlation between this activity count variable and the (primary) upper quartile operationalization was very high $(r=0.94)$ and findings across the models were consistent in direction, magnitude, and statistical significance. Thus, we do not present these analyses. Second, because the wrist-measured physical activity is not expected to mirror more 
commonly used measures of MVPA reported in the literature, a sensitivity analysis was conducted using the MVPA data measured by hip accelerometry at the HCHS/SOL baseline visit as the outcome variable. In addition to the aforementioned covariates, this model additionally controlled for the number of hours/day the device was worn. Third, in analyses of variability in sleep duration or sleep midpoint, we conducted a sensitivity analysis limiting the sample to only participants with a minimum of 7 nights' data $(n=1,862)$, to account for the full range of variability across all days of the week. The effects were consistent in terms of direction, magnitude, and statistical significance to those with the larger sample size, and thus we maintained the larger sample and do not report these analyses further. Finally, we tested the consistency of results across potential effect modifiers of age, sex, and shift work status, which have been shown to modify associations of sleep duration with physical activity in prior research (McClain et al., 2014) or to alter sleep schedules in the HCHS/SOL (Reid et al., 2018). Age, sex, and shift work were entered as product terms separately with sleep duration (both continuous and categorical), sleep midpoint, and variability in sleep duration and midpoint in the models, with physical activity measured by wrist actigraphy as the outcome. Further, to evaluate possible differential effects of sleep variability according to sleep duration, sleep duration (continuous) was entered as a product term with variability in sleep duration and variability in midpoint in separate models.

\section{Results}

A description of the population can be found in Table 1. The mean sleep duration was $6.7(\mathrm{SD}=1.1)$ hours, the mean within-person variability in sleep duration $1.3(\mathrm{SD}=0.6)$ hours, the mean sleep midpoint 04:05:12 ( $\mathrm{SD}=01: 35: 06)$, and the mean within-person variability in sleep midpoint 1.0 $(\mathrm{SD}=0.7)$ hours. The mean number of minutes per day in the upper quartile of physical activity according to concurrent, wrist-worn actigraphy was $250.4(\mathrm{SD}=111.7)$ minutes. The mean number of minutes per day in MVPA according to the baseline hip-worn accelerometer was $26.0(\mathrm{SD}=25.3)$ minutes. The wrist- and hip-worn physical activity measurements (assessed at different points in time) were weakly correlated $(r=0.08)$.

\section{Sleep duration, midpoint, and variability with concurrent wrist-worn actiwatch physical activity}

When analyzed continuously, sleep duration was negatively associated with minutes of physical activity $[\mathrm{B}=-28.7$ ( $\mathrm{SE}=3.8), p<.001$, see model 1 in Table 2] after adjusting for covariates. When variability in sleep duration was added to the model with continuous sleep duration, it was not related to physical activity $[\mathrm{B}=-0.7$ ( $\mathrm{SE}=0.9), p=.43$, see model 2]. When examined categorically, compared to intermediate sleep, short sleep was related to greater minutes of physical activity $[\mathrm{B}=52.3(\mathrm{SE}=9.3), p<.001$, see model 3$]$ and long sleep was associated with fewer minutes of physical activity $[\mathrm{B}=-40.3(\mathrm{SE}=9.4), p<.001]$. Sleep midpoint was not related to physical activity $[\mathrm{B}=-4.6(\mathrm{SE}=2.5), p=.07$, see model 4$]$. When variability in sleep midpoint was added to the model with sleep midpoint, variability in sleep midpoint was positively associated with minutes of physical activity $[\mathrm{B}=24.2(\mathrm{SE}=5.6), p<.001$, model 5].

\section{Sensitivity analyses}

Analyses examining associations of sleep variables with hip-worn accelerometer derived MVPA minutes/day collected at the HCHS/SOL baseline are presented in Table 3. Short sleep duration (reference $=$ intermediate) was associated with greater minutes/day of MVPA $[\mathrm{B}=6.0(\mathrm{SE}=2.5)$, $p=.02$, see model 3 in Table 3]. Variability in sleep duration was related to greater MVPA [B $=4.3$ ( $\mathrm{SE}=1.7), p=.01$, see model 2]. Continuous sleep duration, sleep midpoint, and variability in midpoint were not significantly associated with minutes/day of MVPA. 
Table 1. Demographic, health, sleep, and physical activity characteristics $(\mathrm{N}=2,156), \mathrm{HCHS} / \mathrm{SOL}$ Sueño Ancillary Study (2010-2013).

$\mathrm{n}(\%)$ or $\mathrm{M}(\mathrm{SD})$

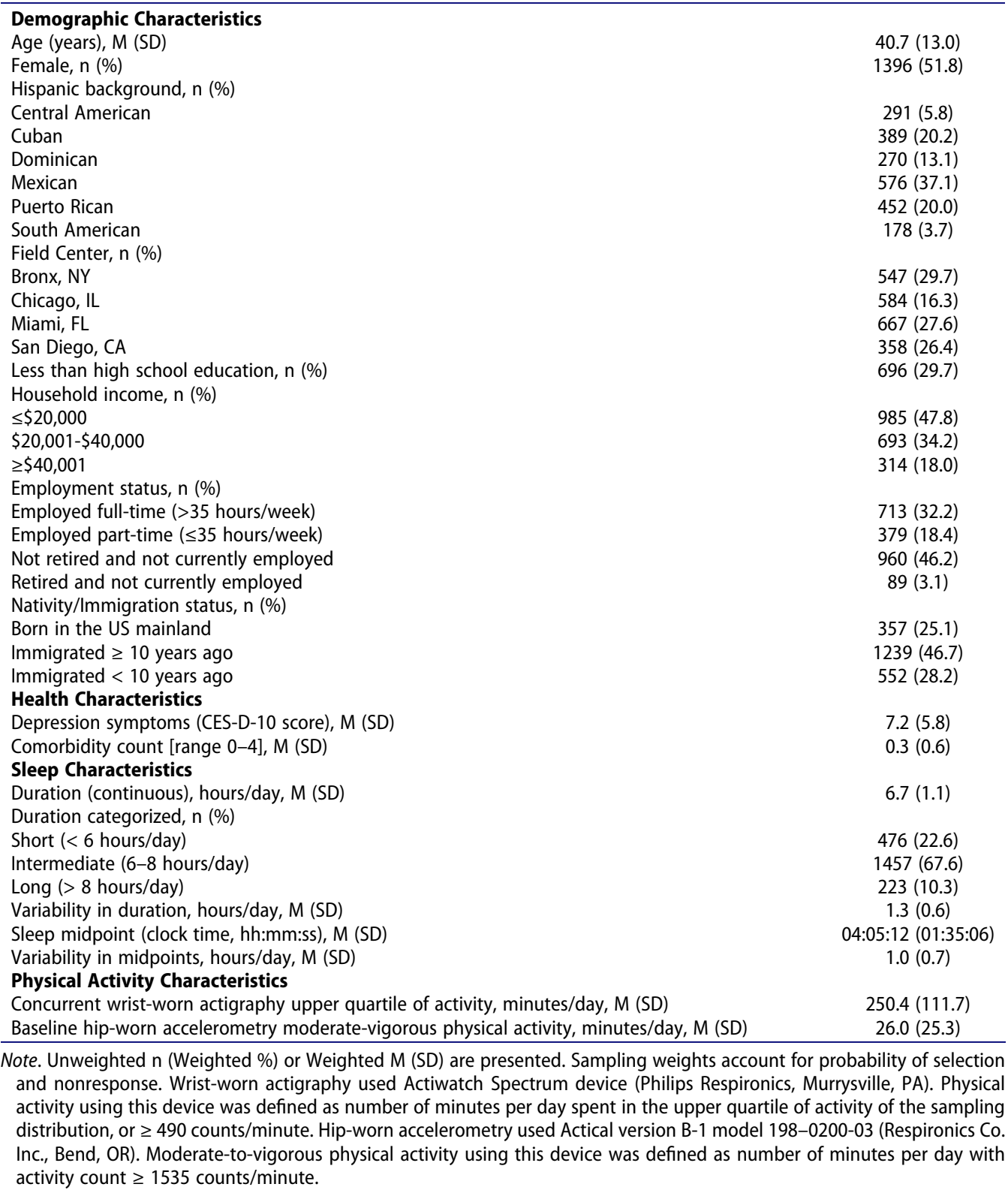

In the effect modification analyses, sex, age, and shift worker status did not moderate associations of sleep variables with minutes/day of Actiwatch-assessed physical activity (all $p>.05$ ). Variability in sleep duration and midpoint also did not moderate the effects of sleep duration in relation to minutes/ day of Actiwatch-assessed physical activity (all $p>.05$ ). 
Table 2. Multivariable linear regression analyses, regressing concurrent wrist-worn Actiwatch physical activity (in minutes) on sleep variables, HCHS/SOL Sueño Ancillary Study (2010-2013).

\begin{tabular}{|c|c|c|c|c|c|}
\hline & Model 1 & Model 2 & Model 3 & Model 4 & Model 5 \\
\hline & $\mathrm{B}(\mathrm{SE})$ & $B(S E)$ & $\mathrm{B}(\mathrm{SE})$ & $B(S E)$ & $\mathrm{B}(\mathrm{SE})$ \\
\hline Sleep duration (hours) & $-28.7(3.8)^{* *}$ & $-37.2(5.2)^{* *}$ & - & - & - \\
\hline \multicolumn{6}{|l|}{$\begin{array}{l}\text { Sleep duration, categorical (intermediate 6-8 = } \\
\text { referent) }\end{array}$} \\
\hline Short sleep ( $<6$ hours) & - & - & $52.3(9.3)^{* *}$ & - & - \\
\hline Long sleep ( $>8$ hours) & - & - & $-40.3(9.4)^{* *}$ & - & - \\
\hline Sleep midpoint (number of hours after 4:00 AM) & - & - & - & $-4.6(2.5)$ & $-6.8(2.4)^{* *}$ \\
\hline Variability in sleep duration, hours/day & - & $-0.7(0.9)$ & - & - & - \\
\hline Variability in sleep midpoint, hours/day & - & - & - & - & $24.2(5.6)^{* *}$ \\
\hline Age, years & $-1.0(0.3)^{* *}$ & $-0.9(0.4)^{*}$ & $-1.0(0.3)^{* *}$ & $-1.1(0.3)^{* *}$ & $-1.1(0.3)^{* *}$ \\
\hline Sex (female $=$ referent) & $-37.6(6.8)^{* *}$ & $-35.7(9.2)^{* *}$ & $-34.8(6.9)^{* *}$ & $-23.8(7.2)^{* *}$ & $-27.2(7.0)^{* *}$ \\
\hline \multicolumn{6}{|l|}{ Field center (Miami = referent) } \\
\hline Bronx & $-15.9(8.8)$ & $-23.8(11.1)^{*}$ & $-15.9(8.9)$ & $-10.3(8.8)$ & $-10.4(8.7)$ \\
\hline Chicago & $20.5(8.0)^{*}$ & $14.8(10.9)$ & $19.3(8.1)^{*}$ & $18.4(8.3)^{*}$ & $19.0(8.3)^{*}$ \\
\hline San Diego & $39.5(11.6)^{* *}$ & $56.3(16.6)^{* *}$ & $40.1(11.9)^{* *}$ & $37.1(12.4)^{* *}$ & $38.0(12.2)^{* *}$ \\
\hline \multicolumn{6}{|l|}{ Household income $(<\$ 20,000=$ referent $)$} \\
\hline$\$ 20,001-40,000$ & $-1.8(8.9)$ & $6.6(11.3)$ & $-0.7(8.9)$ & $-3.7(9.4)$ & $-2.0(9.3)$ \\
\hline$>\$ 40,000$ & $-28.8(9.0)^{* *}$ & $-29.3(12.1)^{*}$ & $-27.7(9.1)^{* *}$ & $-33.4(9.8)^{* *}$ & $-31.1(9.6)^{* *}$ \\
\hline \multicolumn{6}{|l|}{ Employment status (employed full time = referent) } \\
\hline Employed part time & $-16.7(10.4)$ & $-26.7(13.5)^{*}$ & $-14.4(10.0)$ & $-9.8(10.2)$ & $-9.1(10.2)$ \\
\hline Unemployed & $-27.1(83)^{* *}$ & $-35.0(12.1)^{* *}$ & $-28.8(8.3)^{* *}$ & $-31.2(8.4)^{* *}$ & $-28.4(8.1)^{* *}$ \\
\hline Retired & $-34.6(14.1)^{*}$ & $-23.5(19.2)$ & $-30.7(15.5)^{*}$ & $-34.1(17.3)^{*}$ & $-34.1(16.2)^{*}$ \\
\hline Depression (CES-D-10 score) & $-1.3(0.6)^{*}$ & $-0.8(0.8)^{*}$ & $-1.2(0.6)$ & $-1.1(0.6)$ & $-1.2(0.6)^{*}$ \\
\hline Number of Comorbidities & $-11.9(5.5)^{*}$ & $-0.9(6.9)$ & $-11.3(5.5)^{*}$ & $-11.1(5.8)$ & $-9.3(5.8)$ \\
\hline
\end{tabular}

Note: All regression models adjusted for age, sex, field center, household income, employment status, depression symptoms, and comorbid health conditions. Model 1 included continuous sleep duration as an explanatory variable. Model 2 further adjusted model 1 with variability in sleep duration as an explanatory variable. Model 3 included categorical sleep duration as an explanatory variable. Model 4 included sleep midpoint as an explanatory variable. Model 5 further adjusted model 4 and included variability in sleep midpoint as an explanatory variable. ${ }^{*} p<0.05 ;{ }^{* *} p<0.01$.

\section{Discussion}

The current study investigated relationships between objectively measured sleep duration, midpoint, and variability and physical activity among Hispanic/Latino adults representative of four US urban communities using both wrist- and hip-worn devices. Shorter sleep duration (in continuous and categorical analyses) and more variability in sleep midpoint were related to greater minutes of wrist actigraphy-assessed (light to vigorous) physical activity. Sleep midpoint and variability in sleep duration were not significantly associated with wrist actigraphy-assessed physical activity. Sensitivity analyses examining associations of sleep variables with hip-worn accelerometer data from $2.1 \pm$ 0.4 years earlier were generally not statistically significant.

Short and long sleep duration have been shown to be detrimental for health in prior research (Knutson et al., 2007), but in our study short sleep was associated with greater physical activity levels. This result was surprising, considering sleep duration's inverse linear relationship with obesity prevalence and positive linear association with diet quality in the HCHS/SOL Sueño cohort (Loredo et al., 2019; Mossavar-Rahmani et al., 2015, 2017); however, it has been associated with higher levels of self-reported work-related physical activity in the cohort (Tom et al., 2019). When considered in the context of the 24-hr day, the change of time spent in one activity directly influences time spent in another activity (Matricciani et al., 2020). In those obtaining shorter sleep, the extra minutes or hours in the day must be spent engaging in another activity. It is possible that those obtaining shorter sleep might be spending that time on social, occupational, or caregiving activities, which could be augmenting their minutes per day of physical activity (i.e., they are spending less time asleep and more time engaging in active behaviors), which has been shown before among Hispanics/Latinos (Hawkins et al., 2018; Tom et al., 2019). Of the studies that found a positive relationship between sleep duration and physical activity levels, there is evidence that moderating factors such as age and sleep duration may be 
Table 3. Multivariable linear regression analyses, regressing baseline hip-worn accelerometer MVPA (in minutes) on sleep variables, HCHS/SOL baseline visit (2008-2011).

\begin{tabular}{lccccc}
\hline & Model 1 & Model 2 & Model 3 & Model 4 & Model 5 \\
\hline & B (SE) & B (SE) & B (SE) & B (SE) & B (SE) \\
\hline Sleep duration (hours) & $-1.5(0.9)$ & $-1.2(0.8)$ & - & - & - \\
Sleep duration, categorical (intermediate 6-8 = referent) & & & & \\
Short sleep (<6 hours) & - & - & $6.0(2.5)^{*}$ & - & - \\
Long sleep (>8 hours) & - & - & $-0.9(2.4)$ & - & - \\
Sleep midpoint (number of hours after 4:00 AM) & - & - & - & $-0.1(0.6)$ & $-0.2(0.6)$ \\
Variability in sleep duration, hours/day & - & $4.3(1.7)^{*}$ & - & - & - \\
Variability in sleep midpoint, hours/day & - & - & - & - & $0.9(1.0)$ \\
Age, years & $-0.3(0.1)^{* *}$ & $-0.3(0.1)^{* *}$ & $-0.3(0.1)^{* *}$ & $-0.3(0.1)^{* *}$ & $-0.3(0.1)^{* *}$ \\
Sex (female = referent) & $12.7(1.8)^{* *}$ & $12.2(1.7)^{* *}$ & $12.4(1.7)^{* *}$ & $13.4(1.8)^{* *}$ & $13.2(1.8)^{* *}$ \\
Field center (Miami = referent) & & & & & \\
Bronx & $7.5(2.7)^{* *}$ & $7.3(2.7)^{* *}$ & $7.6(2.7)^{* *}$ & $7.3(2.8)^{* *}$ & $7.3(2.8)^{* *}$ \\
Chicago & $1.8(2.4)$ & $1.9(2.4)$ & $2.0(2.4)$ & $1.2(2.4)$ & $1.2(2.4)$ \\
San Diego & $8.9(2.5)^{* *}$ & $8.4(2.3)^{* *}$ & $9.1(2.5)^{* *}$ & $8.8(2.5)^{* *}$ & $8.8(2.5)^{* *}$ \\
Household income (<\$20,000 = referent) & & & & \\
\$20,001-40,000 & $-3.1(2.1)$ & $-2.8(2.1)$ & $-2.7(2.1)$ & $-3.2(2.1)$ & $-3.1(2.2)$ \\
$>\$ 40,000$ & $-7.6(2.2)^{* *}$ & $-6.9(2.0)^{* *}$ & $-7.2(2.2)^{* *}$ & $-7.8(2.2)^{* *}$ & $-7.7(2.2)^{* *}$ \\
Employment status (employed full time = referent) & & & & & \\
Employed part time & $4.5(2.8)$ & $4.0(2.7)$ & $4.5(2.8)$ & $4.8(2.8)$ & $4.8(2.8)$ \\
Unemployed & $-2.7(1.9)$ & $-3.3(1.8)$ & $-2.7(1.9)$ & $-2.9(1.9)$ & $-2.8(1.9)$ \\
Retired & $4.3(5.0)$ & $4.1(5.0)$ & $4.8(5.1)$ & $4.2(4.9)$ & $4.2(4.9)$ \\
Depression (CES-D-10 score) & $-0.3(0.1)$ & $-0.4(0.1)^{* *}$ & $-0.3(0.1)^{*}$ & $-0.3(0.1)^{*}$ & $-0.3(0.1)^{*}$ \\
Number of Comorbidities & $-0.3(1.3)$ & $-0.2(1.3)$ & $-0.5(1.3)$ & $-0.3(1.4)$ & $-0.3(1.4)$ \\
Hours/day accelerometer was worn & $1.9(0.4)^{* *}$ & $1.8(0.4)^{* *}$ & $1.8(0.4)^{* *}$ & $2.0(0.4)^{* *}$ & $1.9(0.4)^{* *}$ \\
\hline
\end{tabular}

Note: All regression models adjusted for age, sex, field center, household income, employment status, depression symptoms, and comorbid health conditions. Model 1 included continuous sleep duration as an explanatory variable. Model 2 further adjusted model 1 with variability in sleep duration as an explanatory variable. Model 3 included categorical sleep duration as an explanatory variable. Model 4 included sleep midpoint as an explanatory variable. Model 5 further adjusted model 4 and included variability in sleep midpoint as an explanatory variable. ${ }^{*} p<0.05 ;{ }^{* *} p<0.01$.

important (McClain et al., 2014). However, the current study found consistent associations of sleep with physical activity across age, sex, and levels of sleep duration. Additionally, some previous research using objective assessment has found no relationship between sleep duration and physical activity (Kline et al., 2017; Mitchell et al., 2016).

In the current study, sleep midpoint did not significantly relate to physical activity levels in primary or sensitivity analyses. These relationships are unclear in the previous research, with some studies showing a relationship between later sleep midpoint and lower levels of physical activity (Shechter \& St-Onge, 2014) and others showing a null association (Kline et al., 2017). Similar to sleep midpoint, those with later bed and wake times were more likely to have less physical activity and be overweight/ obese than those with early bed and early wake times, even though they had similar sleep durations (Olds et al., 2011). Evening chronotype, or a preference to perform daily activities in the evening (vs. daytime), has been associated with less physical activity as well (Haraszti et al., 2014; Makarem et al., 2020). However, our findings do not support that sleep midpoint is associated with physical activity levels in the HCHS/SOL population. While research has linked later sleep timing with higher body mass index (Baron et al., 2012), the association may occur through increased food consumption later in the evening and less nutritious food choices, and not through decreased physical activity (Gariépy et al., 2019).

Of note, greater variability in sleep midpoint related to greater levels of physical activity in the current study. Though the effect magnitude was small, this finding was in the opposite direction of hypotheses based on prior research linking greater variability in sleep timing with less physical activity, using self-report (Duncan et al., 2016; Rutters et al., 2014). With inconsistent sleep timing from night to night, circadian rhythms are disrupted, which has negative physiological consequences for the metabolic, inflammatory, immune, and other physiological systems (Buxton et al., 2012). As posited for those with shorter sleep duration, perhaps individuals who obtain more variable sleep are also more 
active socially and occupationally, which may lead to greater physical activity. In this cohort, shift work schedule has been associated with later sleep timing and more variable sleep (Reid et al., 2018), which may also influence the amount of physical activity obtained. In the current study, the interaction effect of shift work approached significance, but did not reach the cutoff of $p<.05$. In individuals obtaining more variable sleep, the higher levels of physical activity may offset some of the negative physiological consequences of their sleep variability (Saner et al., 2018). Further research is needed to elucidate the effect of sleep variability, in conjunction with physical activity, in individuals at risk of cardiometabolic disease.

In interpreting the findings that shorter sleep duration and greater variability in sleep midpoint related to greater physical activity, it is important to also consider the context through which the physical activity was obtained. In the HCHS/SOL, much of the cohort's physical activity is workrelated (Arredondo et al., 2016), which has been associated with higher mortality in men (Coenen et al., 2018) and overweight/obesity in the HCHS/SOL cohort (Singer et al., 2016). While the objective measure of physical activity has its strengths, with the accelerometer used it misses the ability to characterize the type of physical activity which may provide insight into its relation to health.

While the current study has strengths, such as objective measures of sleep and physical activity in a large and diverse Hispanic/Latino cohort, it also has several limitations. By measuring physical activity using a sensor on the wrist, as opposed to the hip, certain types of physical activity in which the wrist remains relatively inactive may be under-represented. Conversely, the wrist measurement may include activity typically categorized as non-active when measured via the hip, such as typing and fidgeting. Although the use of wrist-worn devices is beginning to gain traction as a means of measuring physical activity (Crouter et al., 2015), the wrist-worn device in the current study has not been widely studied. As such, no MVPA cutoff value has been established for the wrist-worn Actiwatch Spectrum. To obtain contemporaneous data in the current study, a physical activity cutoff was created by using the upper quartile of physical activity from the same device used to measure sleep. The limitation of this approach is that the mean number of minutes/day spent in this category of physical activity is considerably larger than the more standardized MVPA estimates in the cohort and the correlation between the two is very low. This suggests that this operationalization of physical activity includes light intensity physical activity as well as MVPA. Notably, although it is less frequently studied than MVPA, greater light intensity activity as a substitution for sedentary behavior has been linked with lower risk of mortality (Matthews et al., 2015), and the current study contributes new information around total activity (light plus moderate to vigorous). The current study is limited by its cross-sectional design and we are unable to establish temporal associations or mechanisms. Because the hip accelerometer-derived MVPA was collected prior to the sleep data, reverse and bidirectional relationships between these behaviors are possible. Longitudinal studies would be valuable in establishing temporality in these relationships.

In summary, the current study contributes important information about relationships between sleep and physical activity in Hispanics/Latinos, a large and growing US population at risk for low activity levels, and cardiometabolic disorders. By examining multiple objectively measured dimensions of sleep health, the study highlights the importance of intra-individual sleep variability from night to night in sleep timing (i.e., midpoint). In addition to the role of sleep timing in regulating circadian rhythms and other physiological responses, sleep variability may influence physical activity levels. Findings from the current study suggest some possible relationships but raise new research questions. Further experimental or longitudinal research regarding directionality between sleep variability and physical activity is warranted. As both behaviors are modifiable and have been linked with metabolic consequences, conclusive research in this area is needed to guide future recommendations that could aid in prevention of chronic disease. 


\section{Acknowledgments}

The authors thank the staff and participants of HCHS/SOL for their important contributions. Investigators website - http://www.cscc.unc.edu/hchs/

\section{Disclosure statement}

No potential conflict of interest was reported by the authors.

\section{Funding}

This work was supported by the National Heart, Lung, and Blood Institute (NHLBI) under Grants HL098297, HL127307; and author KS was supported by Grant HL079891. The Hispanic Community Health Study/Study of Latinos was carried out as a collaborative study supported by contracts from the National Heart, Lung, and Blood Institute (NHLBI) to the University of North Carolina (N01-HC65233), University of Miami (N01-HC65234), Albert Einstein College of Medicine (N01-HC65235), Northwestern University (N01-HC65236), and San Diego State University (N01-HC65237). The following Institutes/Centers/Offices contribute to the HCHS/SOL through a transfer of funds to the NHLBI: National Center on Minority Health and Health Disparities, the National Institute of Deafness and Other Communications Disorders, the National Institute of Dental and Craniofacial Research, the National Institute of Diabetes and Digestive and Kidney Diseases, the National Institute of Neurological Disorders and Stroke, and the Office of Dietary Supplements.

\section{ORCID}

Kimberly L. Savin (iD) http://orcid.org/0000-0003-1658-1228

\section{References}

2018 Physical Activity Guidelines Advisory Committee. (2018). 2018 physical activity guidelines advisory committee scientific report. U.S. Department of Health and Human Services.

Alcántara, C., Patel, S. R., Carnethon, M., Castañeda, S., Isasi, C. R., Davis, S., Ramos, A., Arredondo, E., Redline, S., Zee, P. C., \& Gallo, L. C. (2017). Stress and sleep: Results from the hispanic community health study/study of latinos sociocultural ancillary study. SSM - Population Health, 3, 713-721. https://doi.org/10.1016/j.ssmph.2017.08.004

Andresen, E. M., Malmgren, J. A., Carter, W. B., \& Patrick, D. L. (1994). Screening for depression in well older adults: Evaluation of a short form of the CES-D (center for epidemiologic studies depression scale). American Journal of Preventive Medicine, 10(2), 77-84. https://doi.org/10.1016/S0749-3797(18)30622-6

Arredondo, E. M., Sotres-Alvarez, D., Stoutenberg, M., Davis, S. M., Crespo, N. C., Carnethon, M. R., Castañeda, S. F., Isasi, C. R., Espinoza, R. A., Daviglus, M. L., Perez, L. G., \& Evenson, K. R. (2016). Physical activity levels in U.S. latino/hispanic adults: Results From the hispanic community health study/study of latinos. American Journal of Preventive Medicine, 50(4), 500-508. https://doi.org/10.1016/j.amepre.2015.08.029

Baron, K. G., Reid, K. J., Kern, A. S., \& Zee, P. C. (2012). Role of sleep timing in caloric intake and BMI. Obesity, 19(7), 1374-1381. https://doi.org/10.1038/oby.2011.100

Bellavia, A., Åkerstedt, T., Bottai, M., Wolk, A., \& Orsini, N. (2014). Sleep duration and survival percentiles across categories of physical activity. American Journal of Epidemiology, 179(4), 484-491. https://doi.org/10.1093/aje/kwt280

Buxton, O. M., Cain, S. W., O’Connor, S. P., Porter, J. H., Duffy, J. F., Wang, W., Czeisler, C. A., \& Shea, S. A. (2012). Adverse metabolic consequences in humans of prolonged sleep restriction combined with circadian disruption. Science Translational Medicine, 4(129), 129ra43. https://doi.org/10.1126/scitranslmed.3003200

Coenen, P., Huysmans, M. A., Holtermann, A., Krause, N., van Mechelen, W., Straker, L. M., \& van der Beek, A. (2018). Do highly physically active workers die early? A systematic review with meta-analysis of data from 193696 participants. British Journal of Sports Medicine, 52(20), 1320-1326. https://doi.org/10.1136/bjsports-2017-098540

Colley, R. C., \& Tremblay, M. S. (2011). Moderate and vigorous physical activity intensity cut-points for the Actical accelerometer. Journal of Sports Sciences, 29(8), 783-789. https://doi.org/10.1080/02640414.2011.557744

Crouter, S. E., Flynn, J. I., \& Bassett, D. R., Jr. (2015). Estimating physical activity in youth using a wrist accelerometer. Medicine \& Science in Sports \& Exercise, 47(5), 944-951. https://doi.org/10.1249/MSS.0000000000000502

Dudley, K. A., Weng, J., Sotres-Alvarez, D., Simonelli, G., Cespedes Feliciano, E., Ramirez, M., Ramos, A. R., Loredo, J. S., Reid, K. J., Mossavar-Rahmani, Y., Zee, P. C., Chirinos, D. A., Gallo, L. C., Wang, R., \& Patel, S. R. (2017). Actigraphic sleep patterns of U.S. hispanics: The hispanic community health study/study of latinos. Sleep, 40 (2), zsw049. https://doi.org/10.1093/sleep/zsw049 
Duncan, M. J., Kline, C. E., Rebar, A. L., Vandelanotte, C., \& Short, C. E. (2016). Greater bed- and wake-time variability is associated with less healthy lifestyle behaviors: A cross-sectional study. Journal of Public Health, 24(1), 31-40. https://doi.org/10.1007/s10389-015-0693-4

Evenson, K. R., Sotres-Alvarez, D., Deng, Y., Marshall, S. J., Isasi, C. R., Esliger, D. W., \& Davis, S. (2015). Accelerometer adherence and performance in a cohort study of US Hispanic adults. Medicine \& Science in Sports \& Exercise, 47(4), 725-734. https://doi.org/10.1249/MSS.0000000000000478

Gabriel, K. P., Sternfeld, B., Shiroma, E. J., Pérez, A., Cheung, J., \& Lee, I.-M. (2017). Bi-directional associations of accelerometer-determined sedentary behavior and physical activity with reported time-in-bed: Women's Health Study. Sleep Health, 3(1), 49-55. https://doi.org/10.1016/j.sleh.2016.10.001

Gariépy, G., Doré, I., Whitehead, R. D., \& Elgar, F. J. (2019). More than just sleeping in: A late timing of sleep is associated with health problems and unhealthy behaviours in adolescents. Sleep Medicine, 56, 66-72. https://doi.org/ 10.1016/j.sleep.2018.10.029

González, P., Nuñez, A., Merz, E., Brintz, C., Weitzman, O., Navas, E. L., Camacho, A., Buelna, C., Penedo, F. J., Wassertheil-Smoller, S., Perreira, K., Isasi, C. R., Choca, J., Talavera, G. A., \& Gallo, L. C. (2017). Measurement properties of the center for epidemiologic studies depression scale (CES-D 10): Findings from HCHS/SOL. Psychological Assessment, 29(4), 372-381. https://doi.org/10.1037/pas0000330

Haraszti, R., Purebl, Á., Salavecz, G., Poole, G., Dockray, L., \& Steptoe, A. (2014). Morningness-eveningness interferes with perceived health, physical activity, diet, and stress levels in working women: A cross-sectional study. Chronobiology International, 31(7), 829-837. https://doi.org/10.3109/07420528.2014.911188

Hawkins, M., Marcus, B., Pekow, P., Rosal, M. C., Tucker, K. L., Spencer, M. C., \& Chasan-Taber, L. (2018). Physical activity and sleep quality and duration during pregnancy among hispanic women: Estudio PARTO. Behavioral Sleep Medicine, 17(6), 804-817. https://doi.org/10.1080/15402002.2018.1518225

Kline, C. E., Krafty, R. T., Mulukutla, S., \& Hall, M. H. (2017). Associations of sedentary time and moderate-vigorous physical activity with sleep-disordered breathing and polysomnographic sleep in community-dwelling adults. Sleep and Breathing, 21(2), 427-434. https://doi.org/10.1007/s11325-016-1434-9

Knutson, K. L., Spiegel, K., Penev, P., \& Van Cauter, E. (2007). The metabolic consequences of sleep deprivation. Sleep Medicine Reviews, 11(3), 163-178. https://doi.org/10.1016/j.smrv.2007.01.002

LaVange, L. M., Kalsbeek, W. D., Sorlie, P. D., Avilés-Santa, L. M., Kaplan, R. C., Barnhart, J., Liu, K., Giachello, A., Lee, D. J., Ryan, J., Criqui, M. H., \& Elder, J. P. (2010). Sample design and cohort selection in the Hispanic Community Health Study/Study of Latinos. Annals of Epidemiology, 20(8), 642-649. https://doi.org/10.1016/j.anne pidem.2010.05.006

Loredo, J. S., Weng, J., Ramos, A. R., Sotres-Alvarez, D., Simonelli, G., Talavera, G. A., \& Patel, S. R. (2019). Sleep patterns and obesity: Hispanic community health study/study of latinos sueño ancillar study. Chest, 156(2), 348-356. https://doi.org/10.1016/j.chest.2018.12.004

Makarem, N., Paul, J., Giardina, E.-G. V., Liao, M., \& Aggarwal, B. (2020). Evening chronotype is associated with poor cardiovascular health and adverse health behaviors in a diverse population of women. Chronobiology International, 37 (5), 673-685. https://doi.org/10.1080/07420528.2020.1732403

Matricciani, L., Paquet, C., Dumuid, D., Fraysse, F., \& Olds, T. (2020). Sleep and physical activity: When a null finding is not really a null finding. Sleep Medicine Reviews, 51, 101302. https://doi.org/10.1016/j.smrv.2020.101302

Matthews, C. E., Moore, S. C., Sampson, J., Blair, A., Xiao, Q., Keadle, S. K., Hollenbeck, A., \& Park, Y. (2015). Mortality Benefits for Replacing Sitting Time with Different Physical Activities. Medicine \& Science in Sports \& Exercise, 47(9), 1833-1840. https://doi.org/10.1249/MSS.0000000000000621

McClain, J. J., Lewin, D. S., Laposky, A. D., Kahle, L., \& Berrigan, D. (2014). Associations between physical activity, sedentary time, sleep duration and daytime sleepiness in US adults. Preventive Medicine, 66, 68-73. https://doi.org/10. 1016/j.ypmed.2014.06.003

Mitchell, J. A., Godbole, S., Moran, K., Murray, K., James, P., Laden, F., Hipp, J. A., Kerr, J., \& Glanz, K. (2016). No evidence of reciprocal associations between daily sleep and physical activity. Medicine \& Science in Sports \& Exercise, 48(10), 1950-1956. https://doi.org/10.1249/MSS.0000000000001000

Morris, C. J., Yang, J. N., Garcia, J. I., Myers, S., Bozzi, I., Wang, W., Buxton, O. M., Shea, S. A., \& Scheer, F. A. J. L. (2015). Endogenous circadian system and circadian misalignment impact glucose tolerance via separate mechanisms in humans. Proceedings of the National Academy of Sciences of the United States of America, 112, E2225-E2234.

Mossavar-Rahmani, Y., Jung, M., Patel, S. R., Sotres-Alvarez, D., Arens, R., Ramos, A., Redline, S., Rock, C. L., \& Van Horn, L. (2015). Eating behavior by sleep duration in the hispanic community health study/study of latinos. Appetite, 95, 275-284. https://doi.org/10.1016/j.appet.2015.07.014

Mossavar-Rahmani, Y., Weng, J., Wang, R., Shaw, P. A., Jung, M., Sotres-Alvarez, D., Castañeda, S. F., Gallo, L. C., Gellman, M. D., Qi, Q., Ramos, A. R., Reid, K. J., Van Horn, L., \& Patel, S. R. (2017). Actigraphic sleep measures and diet quality in the hispanic community health study/study of latinos sueño ancillary study. Journal of Sleep Research, 26(6), 739-746. https://doi.org/10.1111/jsr.12513

Murillo, R., Lambiase, M. J., Rockette-Wagner, B. J., Kriska, A. M., Haibach, J. P., \& Thurston, R. C. (2017). Racial/ethnic differences in the associations between physical activity and sleep duration: A population-based study. Journal of Physical Activity and Health, 14(2), 138-144. https://doi.org/10.1123/jpah.2015-0638 
Muthén, L. K., \& Muthén, B. O. (2006). Mplus. Los Angeles.

Ogilvie, R. P., \& Patel, S. R. (2017). The epidemiology of sleep and obesity. Sleep Health, 3(5), 383-388. https://doi.org/ 10.1016/j.sleh.2017.07.013

Olds, T. S., Maher, C. A., \& Matricciani, L. (2011). Sleep duration or bedtime? Exploring the relationship between sleep habits and weight status and activity patterns. Sleep, 34(10), 1299-1307. https://doi.org/10.5665/SLEEP.1266

Patel, S. R., Weng, J., Rueschman, M., Dudley, K. A., Loredo, J. S., Mossavar-Rahmani, Y., Ramirez, M., Ramos, A. R., Reid, K., Seiger, A. N., Sotres-Alvarez, D., Zee, P. C., \& Wang, R. (2015). Reproducibility of a Standardized Actigraphy Scoring Algorithm for Sleep in a US Hispanic/Latino Population. Sleep, 38(9), 1497-1503. https://doi.org/10.5665/ sleep.4998

Reid, K. J., Weng, J., Ramos, A. R., Zee, P. C., Daviglus, M., Mossavar-Rahmani, Y., Sotres-Alvarez, D., Gallo, L. C., Chirinos, D. A., \& Patel, S. R. (2018). Impact of shift work schedules on actigraphy-based measures of sleep in Hispanic workers: Results from the hispanic community health study/study of latinos ancillary Sueño study. Sleep, 41 (10), 1-8. https://doi.org/10.1093/sleep/zsy131

Rutters, F., Lemmens, S. G., Adam, T. C., Bremmer, M. A., Elders, P. J., Nijpels, G., \& Dekker, J. M. (2014). Is social jetlag associated with an adverse endocrine, behavioral, and cardiovascular risk profile? Journal of Biological Rhythms, 29 (5), 377-383. https://doi.org/10.1177/0748730414550199

Saner, N. J., Bishop, D. J., \& Bartlett, J. D. (2018). Is exercise a viable therapeutic intervention to mitigate mitochondrial dysfunction and insulin resistance induced by sleep loss? Sleep Medicine Reviews, 37, 60-68. https://doi.org/10.1016/j. smrv.2017.01.001

Shan, Z., Ma, H. M., Xie, M., Yan, P., Guo, Y., Bao, W., Rong, Y., Jackson, C. L., Hu, F. B., \& Liu, L. (2015). Sleep duration and risk of type 2 diabetes: A meta-analysis of prospective studies. Diabetes Care, 38, 38(3), 529-537. https://doi.org/ $10.2337 / \mathrm{dc} 14-2073$

Shechter, A., \& St-Onge, M. (2014). Delayed sleep timing is associated with low levels of free-living physical activity in normal sleeping adults. Sleep Medicine, 15(12), 1586-1589. https://doi.org/10.1016/j.sleep.2014.07.010

Singer, R. H., Stoutenberg, M., Gellman, M. D., Archer, E., Davis, S. M., Gotman, N., Marquez, D. X., Buelna, C., Deng, Y., Hosgood, H. D., \& Zambrana, R. E. (2016). Occupational physical activity and body mass index: Results from the hispanic community health study/Study of Latinos. PLoS One, 11(3), e0152339. https://doi.org/10.1371/ journal.pone.0152339

Sorlie, P. D., Avilés-Santa, L. M., Wassertheil-Smoller, S., Kaplan, R. C., Daviglus, M. L., Giachello, A. L., Schneiderman, N., Raij, L., Talavera, G., Allison, M., Lavange, L., Chambless, L. E., \& Heiss, G. (2010). Design and implementation of the Hispanic Community Health Study/Study of Latinos. Annals of Epidemiology, 20(8), 629-641. https://doi.org/10.1016/j.annepidem.2010.03.015

Tom, S. E., Brown, J. P., Avilés-Santa, M. L., Sotres-Alvarez, D., Castañeda, S. F., Patel, S. R., Perreira, K., SanchezJohnsen, L. A. P., Shah, N. A., Zee, P. C., \& Redline, S. (2019). Self-reported sleep duration is associated with time in work physical activity but not transportation or leisure physical activity among Hispanic/Latino adults in the U.S.: Results from the hispanic community health study/Study of latinos. Sleep Health, 6(3), 306-313. https://doi.org/10. 1016/j.sleh.2019.10.001.

Towne, S. D., Ory, M. G., Smith, M. L., Peres, S. C., Pickens, A. W., Mehta, R. K., \& Benden, M. (2017). Accessing physical activity among young adults attending a university: The role of sex, race/ethnicity, technology use, and sleep. BMC Public Health, 17(1), 721. https://doi.org/10.1186/s12889-017-4757-y

Vásquez, E., Strizich, G., Gallo, L., Marshall, S. J., Merchant, G. C., Murillo, R., Penedo, F. J., Salazar, C., Sotres-Alvarez, D., Shaw, B. A., \& Isasi, C. R. (2016). The role of stress in understanding differences in sedentary behavior in hispanic/ latino adults: Results from the hispanic community health study/study of latinos sociocultural ancillary study. Journal of Physical Activity \& Health, 13(3), 310-317. https://doi.org/10.1123/jpah.2014-0608

Wittman, M., Dinich, J., Merrow, M., \& Roenneberg, T. (2006). Social jetlag: Misalignment of biological and social time. Chronobiology International, 23(1-2), 497-509. https://doi.org/10.1080/07420520500545979

Wright, K. P., Drake, A. L., Frey, D. J., Fleshner, M., Desouza, C. A., Gronfier, C., \& Czeisler, C. A. (2015). Influence of sleep deprivation and circadian misalignment on cortisol, inflammatory markers, and cytokine balance. Brain, Behavior, and Immunity, 47, 24-34. https://doi.org/10.1016/j.bbi.2015.01.004 